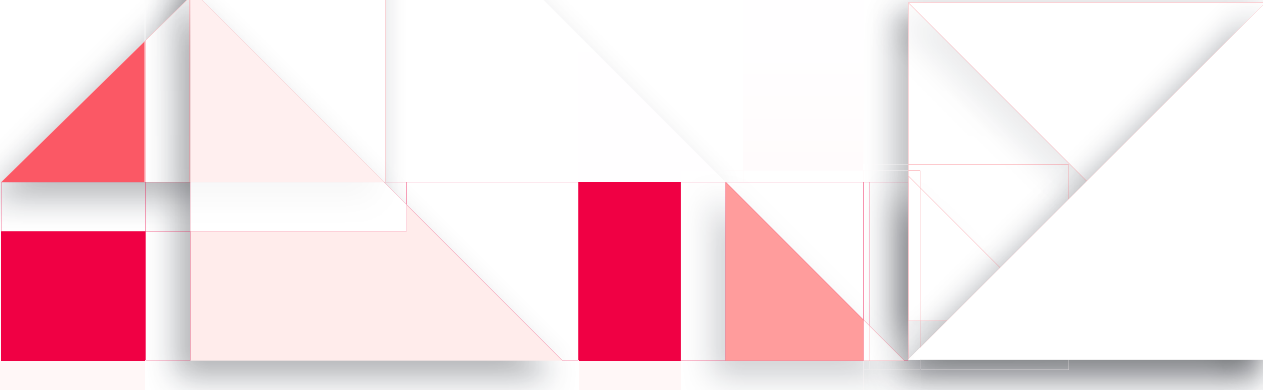

\title{
Imágenes de ciencia en los textos universitarios. Aportes para nuevas retóricas desde la actividad experimental en electrostática
}

- Science Images in University Textbooks. Contributions for New Rhetoric from the Experimental Activity in Electrostatics

- Imagens de ciência nos textos universitários. Contribuições para novas retóricas desde a atividade experimental em eletrostática

\section{Edwin Germán García}

\section{Resumen}

El artículo presenta un estudio de la retórica de los textos universitarios de física fundamental, identificando la yuxtaposición en imágenes de ciencia, problemas conceptuales, ausencia de actividad experimental y coincidencia con explicaciones otorgadas por estudiantes en relación con los significados sobre electrostática. El proyecto se desarrolló en tres fases, primero, la selección de textos objeto de estudio, de acuerdo con el nivel de uso y consulta por parte de profesores y estudiantes universitarios en formación. Segundo, se identificó la retórica presente en el desarrollo discursivo del texto y la actividad experimental asociada a la electrostática, de acuerdo con las técnicas de análisis sugeridas por Van Dijk. Finalmente, se realizó una contrastación entre la retórica de los textos y las explicaciones de los estudiantes a los fenómenos electrostáticos. Se emplearon redes sistémicas sobre narrativas experimentales asociadas a modelos de ciencia, didácticos y de lector.

Se destaca la escasa importancia dada a la actividad experimental y la ausencia del sentido histórico del conocimiento sobre la electricidad en los textos, lo cual, favorece imágenes distorsionadas de la actividad científica y carencia en las explicaciones sobre los fenómenos estudiados. Se analiza la importancia de reflexionar el sentido histórico del conocimiento, la pertinencia de la actividad experimental y el ejercicio de una retórica de construcción de explicaciones y significados asociados a la electrostática.

Palabras clave

Textos universitarios; retórica; actividad experimental; electrostática; propuesta alterna

Doctor en Didáctica de las Matemáticas y las Ciencias Experimentales. Profesor titular de la Universidad del Valle. Cali. Correo electrónico: edwingermangarcia@hotmail.com. Orcid: 0000-0003-1280-667X

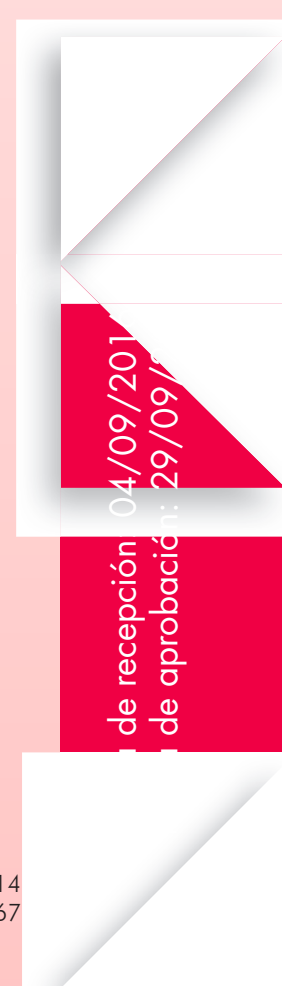




\section{Abstract}

The article presents a study of the rhetoric of fundamental physics university textbooks, identifying the juxtaposition in images of science, conceptual problems, absence of experimental activity, and coincidence with explanations given by students related to the meanings in electrostatics. The project was developed in three phases: first, the selection of the texts under study, according to level of use and consultation by teachers and university students. Second, the identification of the rhetoric present in the discursive development of the text and the experimental activity associated with electrostatics, according to the analysis techniques suggested by Van Dijk. Finally, a comparison was made between the rhetoric of the texts and the students' explanations of the electrostatic phenomena. Systemic networks on experimental narratives associated with science, didactics and reader models were also used.

The article emphasizes the little importance given to the experimental activity and the absence of the historical sense of knowledge about electricity in the texts, which favors distorted images of scientific activity and a lack of explanations about the phenomena under study. It also analyzes the importance of reflecting the historical meaning of knowledge, the relevance of experimental activity, and the exercise of a rhetoric of the development of explanations and meanings associated with electrostatics.

Keywords

University texts; rhetoric; experimental activity; electrostatics; alternative proposal

\section{Resumo}

artigo apresenta um estudo da retórica dos textos universitários de física fundamental, identificando a justaposição em imagens de ciência, problemas conceptuais, ausência de atividade experimental e coincidência com explicações outorgadas por estudantes em relação com os significados sobre eletrostática. $\bigcirc$ projeto foi desenvolvido em três fases: em primeiro lugar, a seleção de textos objeto de estudo de acordo com o nível de uso e consulta por parte de professores es estudantes universitários em formação. Em segundo lugar, foi identificada a retórica presente no desenvolvimento discursivo do texto e a atividade experimental associada à eletrostática, segundo as técnicas de análise sugeridas por Van Dijk. Finalmente, foi realizado um contraste entre a retórica dos textos e as explicações dos estudantes sobre os fenómenos eletrostáticos. Foram empregadas redes sistémicas sobre narrativas experimentais associadas a modelos de ciência, didáticos e de leitor.

Ressalta-se a pouca importância dada à atividade experimental e à ausência do sentido histórico do conhecimento sobre a eletricidade nos textos, que favorece imagens distorcidas da atividade cientifica e insuficiência nas explicações sobre os fenómenos estudados. Analisa-se a importância de refletir sobre o sentido histórico do conhecimento, a pertinência da atividade experimental e o exercício de uma retórica de construção de explicações e significados associados à eletrostática.

Palavras chave

Textos universitários; retórica; atividade experimental; eletrostática; proposta alterna 


\section{Introducción}

El presente documento recoge algunos resultados del proyecto de investigación denominado "Las prácticas experimentales y los libros de texto", para el caso de la enseñanza de las ciencias, biología, química y física, realizado con el apoyo de la Vicerrectoría de Investigaciones de la Universidad del Valle. La propuesta de investigación se desprende de la tesis doctoral del autor en 2011 , donde se considera necesario y urgente un análisis de los textos universitarios, dada su influencia en la imagen de ciencia que apropian los lectores.

Las investigaciones sobre libros de texto no son nuevas (Alambique, 2001 ; Izquierdo, 2005; Perales y Jiménez, 2002) pues estudiantes y profesores en todos los niveles de escolaridad los usan. Lo que sí es nuevo es el enfoque que relaciona la retórica con la actividad experimental (García, 2011 ).

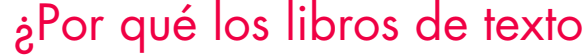 universitarios?}

La triada del sistema didáctico: profesor, estudiante y contenido ha sido ampliamente documentada en didáctica de las ciencias, y es mucho lo que se ha avanzado en comprender su funcionamiento. La relación enseñanza-aprendizaje es su fundamento en los libros de texto (Del Carmen y Jiménez, 1997; Otero, 1990); sin embargo, el tema del "contenido" no parece sufrir modificaciones en las investigaciones realizadas. La preocupación por los contenidos que se van a enseñar no ha sido relevante; los investigadores en lugar de enfocarse en qué contenidos se deben enseñar, se han inclinado a investigar cómo enseñarlos (Calderero, 2003). Es que la palabra contenidos parece estar resuelta desde los propios textos, pues los de física general, química fundamental y biología general de uso frecuente en las universidades como apoyo a la enseñanza no han sufrido modificaciones sustantivas en sus contenidos (aunque transformen la imagen didáctica del mismo), y siguen siendo objeto de consulta amplia por parte de estudiantes y profesores.

De igual manera se encuentra que las investigaciones realizadas se han centrado en la formación teórica del alumno (aprendizaje de leyes y teorías) y han prestado poca atención a la formación práctica (conocimiento de instrumentos, medidas y prácticas experimentales), lo que coincide con la presentación teórica y poco experimental que hacen los textos. Esto nos sugiere preguntas como ¿̇Qué imagen de ciencia transmiten los textos a sus lectores? ¿Qué concepciones existen sobre las prácticas experimentales? ¿Qué tipo de prácticas experimentales se consideran en los textos? ¿Perciben los profesores la necesidad de modificar los contenidos y estrategias de enseñanza de las ciencias atendiendo a la actividad experimental?

\section{La narrativa experimental}

En el caso del estudio de los libros de texto, se han hecho redes sistémicas para obtener información relacionada con diversos enfoques y propositos; estructura semántica (argumentaciones), sintáctica (conceptos presentes), simbólica (ilustraciones presentes), curricular (tipos de actividades) y evolutiva (grado de dificultad) (Perales y Jiménez, 2002). En nuestro caso particular la presencia de lo que Paolo Guidoni et al. (1990) denominan "una buena retórica" que contribuya a desarrollar relaciones de coherencia entre el conocimiento (lo que se piensa), la intervención en los fenomenos (lo que se hace) y el lenguaje (lo que se dice) (Izquierdo, 2005a; Izquierdo y Ribera, 1997).

Dentro de las redes sistémicas existentes hemos optado por una que recoge el interés de 
este trabajo y es la propuesta realizada por el grupo Llenguatge i Ensenyament de les Ciències (LIEC) de la Universidad Autónoma de Barcelona. Este grupo identificó estilos retóricos en libros de texto (Izquierdo et al. 2005a) haciendo referencia a modelos de ciencia, modelos de lector, modelo didáctico y factualidad, esto es los hechos que se describen en el texto.

En una red sistémica como la que se propone (véase la tabla 1) es posible seleccionar y organizar la información cualitativa, teniendo en cuenta las categorías como "criterios o indicadores que permiten caracterizar las diferentes 'narrativas experimentales' que se repiten en diferentes textos con una función específica" (Izquierdo et al., 2005).

Tabla 1. Narrativas experimentales

\begin{tabular}{|c|c|c|c|}
\hline \multirow{12}{*}{ 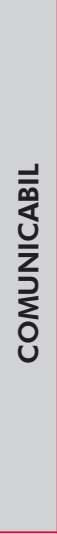 } & \multirow[t]{4}{*}{ Modelo de ciencia } & \multirow[t]{2}{*}{ Dogmático, afirmativo } & Retórica apodíctica \\
\hline & & & Retórica magistral \\
\hline & & \multirow[t]{2}{*}{ Resolución de duda } & Duda retórica \\
\hline & & & Duda real \\
\hline & \multirow[t]{4}{*}{ Modelo didáctico } & \multirow[t]{3}{*}{ Coherente } & Transmisivo \\
\hline & & & Constructivista \\
\hline & & & Descubrimiento \\
\hline & & Incoherente & Incoherente \\
\hline & \multirow[t]{4}{*}{ Modelo de lector } & \multirow[t]{2}{*}{ Didáctica tradicional } & Discípulo \\
\hline & & & Colega \\
\hline & & \multirow[t]{2}{*}{ Nueva didáctica } & Colaborador \\
\hline & & & Aprendiz activo \\
\hline & \multirow[t]{3}{*}{ Fenómenos que narra } & \multirow[t]{2}{*}{ Hechos reales } & Vida cotidiana \\
\hline & & & Laboratorio \\
\hline & & \multicolumn{2}{|c|}{ Hechos transformados, símbolos } \\
\hline
\end{tabular}

\section{Retórica de los textos universitarios: el caso de la electrostática}

Para comprender el problema de la retórica presente en los libros de texto universitarios, se hizo un estudio detallado en torno a una temática particular, la electrostática, y se contrastó con las imágenes que los estudiantes ponen en evidencia cuando dan explicaciones a los fenómenos físicos. Se identificaron las deficiencias conceptuales, como lo sugiere Furió (1997). El estudio de los libros se realizó en dos etapas, de acuerdo con los siguientes criterios.

\section{Etapa de exploración}

Inicialmente se hizo la revisión en bibliotecas universitarias, con profesores y estudiantes sobre los libros de texto de biología, química y física general más consultados y utilizados por los estudiantes o por los profesores en sus clases. Posteriormente se hizo una selección de los textos más representativos (de cada disciplina) y se aplicaron los instrumentos mencionados para obtener información. 
En esta etapa pudimos identificar cuatro fases, a saber: (a) levantamiento bibliográfico de libros, (b) definición de criterios para la selección de textos por intervenir, (c) selección de estudios de caso (capítulos de libros) apropiados para aplicar instrumentos, y (d) aplicación de instrumentos a textos seleccionados en pruebas piloto, reestructuración del instrumento y aplicación definitiva.

\section{Etapa de sistematización}

Comprendió la aplicación de los instrumentos de observación (ajuste y precisión), recolección, sistematización y análisis de resultados. Esta etapa comprende cuatro fases: (a) identificación de la retórica presente en los textos analizados, (b) determinación de las concepciones de prácticas experimentales identificadas en ellos, (c) contrastación y discusión de las concepciones caracterizadas por el estudio, y (d) divulgación de los resultados.

\section{Población y muestra}

Se seleccionó un grupo de 16 estudiantes de último semestre de Licenciatura en Ciencias Naturales y Educación Ambiental, quienes respondieron un cuestionario sobre electrostática, para identificar sus modelos mentales explicativos en torno al fenómeno (Moreira et al., 1998). Se pedía como requisito para contestar el cuestionario haber cursado y aprobado los cursos de física general.

De una muestra de veinte libros de física de los más utilizados comercialmente en Iberoamérica, y dado que el ejercicio que se plantea es de seguimiento riguroso a la retórica empleada, se escogieron los tres de mayor circulación, según fuente de las bibliotecas universitarias. También se tuvo en cuenta que fueran libros utilizados en las clases de física por los estudiantes, de tal manera que se pudieran establecer conexiones entre ellos. Los textos son los siguientes libros de física general dirigidos a estudiantes de primeros semestres de universidad:

Tipler, P. A. y Mosca, G. (2005). Física para la ciencia y la ingeniería (vol. II). Barcelona: Editorial Reverte.

Sears, F. W.; Zemansky, M. W. y Young, H. D. (1986). Física universitaria. EeUU: Editorial Fondo Educativo Interamericano.

Alonso, M. y Finn, E. (1987). Física (vol. II). Campos y ondas. EeUU: Addison-Wesley Iberoamericana.

\section{Resultados en física}

\section{Microestructura del texto}

De acuerdo con el índice, identificamos los siguientes aspectos fundamentales en la presentación del capítulo:

1. La pregunta de introducción al capítulo, "¿Cuál es la carga total de los electrones de una moneda?" da a entender que el modelo expositivo es sobre sistemas discretos de carga. Se inicia el capítulo con un experimento de laboratorio sobre frotación entre barras de plástico y de vidrio, para mostrar la existencia de atracción y repulsión eléctrica; sin embargo no se menciona el objeto con que se frotan los cuerpos considerados.

2. Luego, se recurre a experiencias de Benjamin Franklin para clasificar los materiales y justificar la serie triboeléctrica "los que se cargan como el vidrio frotada con un paño de seda y los que se cargan como el plástico, frotado con un trozo de piel". Además, ponen en evidencia que los 
materiales adquieren carga eléctrica y que "los objetos que portan el mismo tipo de carga se repelen entre sí, mientras que los que portan cargas opuestas se atraen mutuamente". Aquí se da una primera explicación que parte de un modelo newtoniano de partículas ("cuando un vidrio se frota con un trozo de seda, se transfieren electrones del vidrio al pedazo de seda") y cuya información suele ser recurrente entre los estudiantes.

3. De acuerdo con el modelo atómico de la materia, se afirma la existencia de electrones, protones y neutrones y la condición eléctrica de los mismos, para justificar la unidad fundamental de carga e asociada a dichas partículas y, así, introducir la idea de que la carga está cuantizada en un factor $\mathrm{Ne}$ donde $\mathrm{N}$ es un número entero.

4. A partir de la frotación de los objetos se afirma que "uno de ellos queda con un número en exceso de electrones y se carga negativamente y el otro con un déficit de electrones y su carga es positiva". Posteriormente, sin ninguna experiencia o comentario adicional, se afirma que "la carga, suma de los dos objetos, no cambia", lo que se conoce como una ley fundamental de la naturaleza, ley de conservación de la carga. Se define la unidad de carga y se registra el valor de la unidad fundamental de carga e.

5. Se definen directamente conductores y aislantes, sin experiencia alguna que haga referencia a los mismos y básicamente a partir del movimiento de electrones "en los metales pueden moverse libremente, estos materiales se denominan conductores, y en la madera o el vidrio no pueden moverse libremente, estos materiales se denominan aislantes". A continuación se da una explicación del comportamiento de los electrones en los átomos en los metales desde la física atómica. Finalmente se explica el comportamiento del electroscopio desde la noción de transferencia de electrones.

6. Para explicar la inducción eléctrica, el texto parte de un experimento de dos esferas metálicas y una barra cargada. Hay un dibujo que muestra cómo las cargas eléctricas se distribuyen en las esferas por la proximidad de la barra cargada (movimiento de electrones), y se obtiene una carga positiva o negativa según sea el caso, "si la barra se retira y las esferas se separan, éstas quedan uniformemente cargadas con cargas iguales y opuestas". La alusión al comportamiento de la Tierra como conductor y a la inducción por conexión a Tierra se hace con el mismo experimento de las esferas, pero ahora se coloca una de ellas en contacto a Tierra y se "observa" que "al quitar la barra, la esfera queda cargada negativa y uniformemente". La presentación se hace desde el modelo de partículas cargadas, específicamente los electrones. No se reconocen otro tipo de experiencias sobre la inducción eléctrica. 
7. Luego se introduce la ley de Coulomb, a partir de una referencia histórica de Charles A. Coulomb (1736-1806) utilizando una balanza de torsión, sin mencionar cómo funciona la balanza o qué fue lo que el científico hizo con su balanza. Solo menciona que "utilizó el fenómeno de inducción", lo que no deja de ser contradictorio ya que precisamente la inducción eléctrica no tiene explicación desde el modelo newtoniano. También se afirma que "las cargas podían considerarse como puntuales" para producir esferas igualmente cargadas, es decir cargas cuantitativamente idénticas y que al final obtuvo lo que se traduce ahora como la ley de Coulomb. Se escribe la definición de la ley tanto en forma descriptiva como matemática (utilizando vectores). Se establece una semejanza entre esta y la ley de Newton de la gravedad, para mostrar que "ambas dependen de la inversa del cuadrado de la distancia", pero la segunda es solo atractiva. En adelante el texto presenta una serie de ejemplos para hacer cálculos de fuerza eléctrica, ya sea en un átomo "hidrógeno" o en un sistema de cargas, todas ellas utilizando vectores.

8. Es interesante mostrar cómo a pesar de la presentación expositiva afirmativa que se ha hecho de la electrostática en el texto, ahora los autores introducen el campo eléctrico a partir de un problema: "¿̇cuál es el mecanismo según el cual una partícula puede ejercer una fuerza sobre otra a través del espacio vacío que existe entre las partículas?". Si bien la pregunta es compleja, más aún porque se considera en condiciones de vacío, sí sugiere una necesaria presencia del espacio entre ellas, esto es, el campo. Pero lo hacen para eliminar el problema de la visión newtoniana del sistema de partículas, y es el hecho de la "acción a distancia". El campo, ahora, es una creación de la carga, que resulta ser activo, pues "una carga crea un campo eléctrico $E$ en todo el espacio y este campo ejerce una fuerza sobre la otra carga". Sin embargo, esta afirmación resulta incómoda para los autores, ya que a lo largo del capítulo han insistido en la fuerza eléctrica de las partículas cargadas. Para superar este "obstáculo" se afirma ahora que "la fuerza es así ejercida por el campo E existente en la posición de la segunda carga, más que por la propia primera carga que se encuentra a cierta distancia" lo que les permite "salvar" la primera carga, pero... la contradicción en el texto entre acción contigua de campo y acción (fuerza eléctrica) a distancia de partículas cargadas se mantiene. Enseguida se hace una afirmación que para un lector atento no deja de ser desconcertante "los cambios del campo se propagan a través del espacio con la velocidad de la luz, $c^{\prime \prime}$. Esto sugiere un campo activo tal como lo estableció Faraday, pero no aclara nada respecto a la velocidad de la luz, c. En lo que sigue de la presentación se introduce ahora la llamada carga testigo $\left(q^{\circ}\right)$ y se hace una presentación operativa, donde ahora "el campo eléctrico $E$ en un punto se define por esta fuerza dividida por $q^{\circ \prime \prime}$.

9. Ya definido el campo de forma operatoria, se presentan las unidades de 
medida y una tabla que muestra "algunos campos eléctricos de la naturaleza, información descontextualizada y al margen de la problemática presentada. Los ejercicios que se presentan en adelante son para hacer cálculos de campos eléctricos, fuerzas eléctricas o cargas eléctricas en situaciones diferentes, conservando el sistema vectorial y aumentando el nivel de complejidad matemática.

10. Se define el dipolo eléctrico y se presenta la fórmula matemática del momento bipolar eléctrico. Luego se presenta matemáticamente el campo eléctrico en función del momento bipolar. Ahora se afirma que el campo eléctrico $E$ decrece en función del inverso del cubo para un dipolo, pero se reitera que "el decrecimiento con el inverso del cuadrado es para grandes distancias". Lo preocupante es que en los pasajes anteriores se había mencionado que el inverso del cuadrado se había establecido con un experimento entre cargas muy próximas (balanza de torsión) y su validez tenía la legitimidad de ser ley de la naturaleza. Ahora las excepciones resultan confusas, porque no muestran los límites de lo que se quiere decir con "grandes distancias".

11. Atención especial dedican los autores a la presentación de las líneas de campo eléctrico, las cuales son representaciones del campo, ya que "un campo puede representarse dibujando líneas que indiquen su dirección". Como es una representación, el problema se reduce a dibujar líneas de fuerza y expresar matemáticamente el número de entradas y salidas de las cargas puntuales, tanto para una o más cargas, de signos iguales o contrarios, cerca o lejos, en dipolos... con fotos y dibujos se ejemplifica la presencia de líneas de fuerza. Se presentan las "reglas para dibujar líneas de campo eléctrico" y se procede a considerar situaciones diversas para hacer cálculos de líneas de campo. Después, y como si los autores identificaran un cabo suelto afirman que "la relación establecida entre la intensidad del campo eléctrico y las líneas de campo eléctrico es válida" sin hacer alguna otra consideración que permita comprender la dimensión de tal afirmación. Se recurre nuevamente a una analogía con el campo gravitatorio para dar cuenta del decrecimiento con el inverso del cuadrado. No se menciona la importancia de las líneas para la comprensión del electromagnetismo desde la visión de campos, ni se tiene en cuenta la inconsistencia entre las líneas de campo y la visión de acción recta en el sistema de partículas newtoniano.

12. El capítulo termina con análisis matemáticos de movimiento de cargas eléctricas o dipolos eléctricos en campos eléctricos, ya sea paralelos o perpendiculares a la dirección del movimiento. También se presentan ejemplos de aplicación industrial en fotocopiadoras o televisores, hornos de microondas y otros aparatos. Se hace énfasis en el estado de polarización de una partícula y se explica cuándo una molécula es polar. 
Se presenta un resumen del capítulo y una serie de problemas para que el estudiante resuelva.

\section{Comparación de las problemáticas presentes en textos y estudiantes}

A partir de las dificultades evidenciadas en las explicaciones de los estudiantes, como lo sugieren Ogborn, Kress y Martins, I. (1998), en el cuestionario y del análisis de la estructura retórica de los libros de texto y la secuencia del contenido relacionado con la electrostática, de acuerdo con Solbes y Furio (2005) podemos considerar los siguientes aspectos:

\section{Sobre la electrificación (carga eléctrica)}

Debemos empezar por considerar que las preguntas del cuestionario hacen referencia a la electrificación en términos de la capacidad de poner en evidencia la virtud eléctrica de los materiales. Los libros de texto analizados se refieren a la carga eléctrica como una propiedad dada de la materia, "un cuerpo que está electrizado quiere decir que posee carga eléctrica o bien está cargado eléctricamente".

Lo deseable desde el cuestionario aplicado es que los estudiantes den cuenta de que el comportamiento eléctrico depende de los materiales $u$ objetos utilizados, el material frotado y el material con que se frota, y no de la fuerza con que se frotan los mismos. Si comprenden la triboelectricidad, pueden caracterizar el comportamiento de los materiales (incluso como una afinidad para captar electrones); sin embargo, el resultado del cuestionario pone en evidencia que no lo comprenden. Las dificultades en las explicaciones de los estudiantes están arraigadas en el modelo de partículas, en el cual el comportamiento eléctrico de la materia se explica en términos de movimiento y transferencia de electrones.

Los libros de texto analizados coinciden en caracterizar la carga eléctrica desde el modelo atómico y dan cuenta de la electrificación como un problema de transferencia de electrones. No hacen referencia al comportamiento de los materiales, no explican por qué se suele utilizar vidrio, resina, ebonita o ámbar en los experimentos, más que otros materiales. Solamente un libro hace referencia a la serie triboeléctrica, pero más como un problema de ubicación de los materiales "por su afinidad para captar electrones". Además, aunque lo hace a través de una tabla llamada "Serie triboeléctrica", no reconoce el material con el que han sido frotados los materiales, aspecto fundamental de la triboelectricidad.

Podemos entonces considerar que:

- Los estudiantes no comprenden la triboelectricidad, ni los libros de texto estudiados la explican.

- Los estudiantes centran las explicaciones de la electrificación en procesos de transferencia de electrones, en tanto que los libros estudiados tienen en común el abordar la carga eléctrica en términos de transferencia de electrones, desde la estructura eléctrica de la materia y desde el modelo atómico de la materia. Ninguno trata el problema como comportamiento del medio, propio de la visión de campos.

- los estudiantes no consideran límites al flujo de electrones en un material frotado. En muchas de las respuestas se aprecia que cuanto más se froten los materiales, mejor efecto de atracción producen, porque mayor número de electrones se transfieren. Los libros de texto no hablan de límites 
en la "afinidad para captar electrones" o de la "transferencia de electrones de un material a otro"; la experiencia se acomoda al modelo de explicación que ofrecen, sin tener en cuenta el conocimiento cotidiano ni recurrir a experiencias históricas sobre la triboelectricidad.

\section{Sobre la conservación de la electrificación (ley de conservación de la carga)}

J. C. Maxwell en su Tratado de electricidad y magnetismo (1951) propone demostrar con los electroscopios que el medio tiene que ver en los efectos observados. La condición eléctrica es un estado del medio y no se centra en los cuerpos. Los estudiantes tienen dificultad para comprender el comportamiento del medio, por eso consideran en sus explicaciones acciones a distancia entre cuerpos cargados, o movimientos de electrones que chocan y se anulan. Los libros estudiados limitan el tema a la definición del principio o ley de conservación de carga como exceso o déficit de electrones, cuya suma da la carga total que debe ser siempre la misma. Los textos suelen hacer referencia al principio de conservación en términos de que la carga no se crea ni se destruye, solo se transforma; pero igualmente consideran dos clases de electricidad, la positiva y la negativa. No se manifiesta en absoluto el problema planteado por Franklin sobre la cantidad de electricidad.

Es claro que en todos los textos analizados no se asume como problema fundamental el comportamiento del medio. Solo en uno de ellos se hace referencia a un problema que involucra el comportamiento eléctrico de una partícula en el espacio, pero la explicación termina por mostrar el campo como una creación de la carga e incluso con capacidad de actuar en las partículas vecinas, mientras que el poder de la acción se mantiene en "el cuerpo cargado" o en la partícula con carga inicial, contradicción puesta en evidencia en el análisis del texto. En adelante los libros estudiados asumen el campo como un ente matemático y dedican sus páginas a resolver ejercicios. Podemos entonces concluir que:

- Los estudiantes no consideran el comportamiento del medio, ni acuden al campo eléctrico para explicar el comportamiento de los electroscopios. En los textos analizados el campo eléctrico se toma como una representación matemática sin incidencia en las explicaciones de conservación de carga.

- Los estudiantes piensan que las cargas se anulan por acción a distancia o por el movimiento de los electrones. Los libros textos definen la conservación de la carga en términos del número total de electrones en un material y no hacen referencia a experiencia alguna que se identifique con la definición. 
Sobre la comunicación de la electrificación

El conocimiento sobre los conductores y aislantes es muy recurrente en la enseñanza de la electrostática; sin embargo, hay dificultades en la forma como los estudiantes creen que se comunica la electrificación. La comunicación se asume desde el movimiento de cargas eléctricas; cuando se frota un material los electrones se mueven de una región a otra. La dificultad se hace evidente en que todo se vuelve movimiento de cargas, incluso en los dieléctricos, en los que los electrones son arrastrados desde una región a otra. No diferencian los conductores de los aislantes por las características de los materiales, sino por los electrones que portan.

En los libros analizados reducen el asunto a considerar que "algunos materiales permiten el movimiento de carga de una parte del material a otra, mientras que otros no lo permiten". Este tipo de afirmaciones que se centran en el movimiento de carga, no tienen en cuenta la importancia de la organización de los materiales. Se asume como conductor al que permite el movimiento de cargas y aislante al que no:

... en muchos materiales tales como

el cobre y otros metales, parte de los electrones pueden moverse libremente en el seno del material, estos se denominan conductores, y otros materiales como la madera o vidrio, todos los electrones están ligados a los átomos y ninguno puede moverse libremente, denominados aislantes.

La historia de la física ha mostrado que este tipo de afirmaciones son contrarias a la realidad, pues todo material permite el movimiento de cargas, ya sea muy pequeño o muy alto dependiendo de las características de los materiales. Además, desde este tipo de afirmaciones es complicado pensar en semiconductores.

Otro aspecto que se deriva del análisis de los textos es el tipo de experimentos realizados. Decir que un material es conductor porque es metal y dieléctrico porque es vidrio no tiene mayor significación para el estudiante. El electroscopio se utiliza como único referente para justificar cuando la carga pasa a través de un conductor metálico. Este ejemplo es típico de un experimento acomodado a la definición, ya que coincide exactamente con lo que se afirma, pero si alguien preguntara ¿̇qué se puede decir entonces del aire? el libro noofrece elementos para responder.

Se puede considerar entonces que los estudiantes presentan una imagen confusa del movimiento de los electrones. Por una parte, se pueden mover (debido a la frotación) de un lugar a otro; por la otra, no se pueden mover por el tipo de material en el que están, pero conservan la carga. Por lo tanto, las respuestas asociadas al comportamiento de los dieléctricos están ajustadas a una definición que no brinda elementos para contestar la pregunta del cuestionario. Podemos entonces concluir que:

- Los estudiantes no comprenden la comunicación de la electrificación, no caracterizan los materiales y tampoco entienden los conceptos de materiales dieléctricos y conductores. Los textos solamente abordan la comunicación de la carga como el proceso de transferencia de electrones, esto es, conductores y aislantes.

- Los estudiantes solamente explican los fenómenos en términos de movimiento de electrones, lo que evidencia su dificultad para dar cuenta de situaciones diferentes. Los libros no presentan 
experiencias para mostrar la relación entre conductores y aislantes, solo exponen definiciones.

\section{Sobre la inducción eléctrica}

La inducción eléctrica es un fenómeno eléctrico descubierto por Faraday, que permitió el desarrollo del electromagnetismo. Es un fenómeno que no se puede explicar desde el modelo newtoniano de acción a distancia y en línea recta. Para explicarlo, fue necesario acudir al modelo de acción contigua, allí surge la noción de campo eléctrico. Los estudiantes tienen la falsa creencia de que existen dos formas diferentes para electrificar los objetos, por inducción y por conducción (García, 1999). En principio ellas parecen excluyentes: la inducción es a distancia y la conducción por contacto; a medida que el cuerpo electrificado se aleja, la acción inductiva disminuye y a medida que se acerca, aumenta. En cambio, si el cuerpo electrificado toca el otro objeto (electroscopio) le transfiere la carga por conducción. La conducción implica permanencia de la carga y la inducción no. Las respuestas de los estudiantes al cuestionario muestran dificultad en que no pueden identificar cuándo hay inducción y cuándo hay conducción, dado su esquema. La experiencia de Faraday muestra que el objeto se puede mover libremente dentro del recipiente metálico, sin embargo la lectura del electroscopio no cambia, permanece. Esto puede parecer un caso de conducción eléctrica, pero no es así. Faraday considera que no es posible diferenciar clara y distintamente cuándo una acción es inductiva y cuándo no. Los libros muestran cómo al acercar un objeto a otro le transmiten carga por inducción (en algunos casos utilizan esferas) "al acercar a una de las esferas una barra cargada, los electrones fluyen de una esfera a la otra, acercándose a la barra si esta se encuentra positivamente cargada o alejándose si su carga es negativa". Esta presentación hace referencia a la proximidad de la barra y no al contacto, además recurren al contacto con tierra para producir otro efecto inductivo al que llaman "inducción por conexión a tierra". En concordancia con las interpretaciones de los textos en términos de movimiento de electrones, si hay movimiento por transferencia de electrones entonces hay conducción, pero si no lo hay entonces es inducción. De acuerdo con Faraday, como el fenómeno se debe a una acción contigua, no considera movimientos por transferencia de electrones. Los estudiantes no reconocen la acción contigua.

Los textos analizados muestran una yuxtaposición de imágenes y explicaciones sobre los fenómenos estudiados. Presentan el modelo newtoniano de acción a distancia y lo yuxtaponen con el modelo de acción contigua de Faraday, pero sin reparar en que son dos modelos epistemológicamente diferentes y con formas de explicar el mundo también diferente. La única coincidencia está en las formas matemáticas que utilizan. Los libros de texto confunden al estudiante y no le permiten tener claridad respecto a los fenómenos estudiados y los modelos explicativos utilizados. 
Sobre la cuantificación de la electrificación (cuantificación de la carga)

Uno de los aspectos centrales del electromagnetismo asociado a la noción de carga es la medida. El propio experimento de Coulomb fue posible porque consideró la cuantificación de la carga; los de Maxwell permiten establecer claramente la cuantificación de la electrificación e incluso tomarla como unidad de medida. Gracias a los experimentos de Millikan se obtuvieron valores de unidad de carga para el electrón. El alto índice de estudiantes que no reconocen experimentalmente nada sobre cuantificación de la carga refleja su desconocimiento de la electrostática, los que vagamente recuerdan o asocian con palabras como voltímetro o amperímetro es porque piensan que algo tiene que ver con la medida, pero no tienen claridad al respecto. ¿Por qué los estudiantes no reconocen ni referencian la cuantificación de la carga? Los libros estudiados la explican, e incluso algunos avanzan en toda una caracterización del carácter discreto de la materia y de su medida. La información está en los textos: "la carga eléctrica aparece no en cualquier cantidad, sino en múltiplos de una unidad fundamental o cuanto". Es importante anotar que la noción de cuantificación que aparece en los libros se aleja significativamente de la propuesta por Maxwell en su teoría electromagnética, puesto que los experimentos que realiza lo llevan a considerar la carga como un estado del medio y es precisamente ese estado el que se puede cuantificar, no hay un carácter ontológico de la carga. Los estudiantes no apropian la información de los textos sobre la cuantificación de la carga porque no resulta relevante para ellos.
Aportes para una propuesta alterna basada en la actividad experimental

Si bien las experiencias más cercanas sobre electrostática que tenemos están relacionadas con la frotación entre dos objetos, poco o nada se conoce sobre el comportamiento eléctrico de materiales particulares al ser frotados. Experiencias comunes como atraer papelitos, observar chispas cuando una persona se quita una prenda de seda, sentir chispas en la pantalla del televisor cuando está encendido, son algunos ejemplos que hacen referencia a la electrificación. La experiencia más antigua y cercana sobre el tema es la frotación de los cuerpos (retomando así los planteamientos y problemáticas propuestas por W. Gilbert). Cuando se frotan dos objetos entre sí, se establece que ellos evidencian electrificación porque adquieren la virtud o capacidad de atraer otros objetos más pequeños y sensibles que se encuentren en su vecindad. Consideramos entonces la exploración del comportamiento eléctrico de los materiales a partir de la frotación; la planteamos, pues, como el centro de actividades desde el cual iniciar la organización y ampliación del fenómeno eléctrico.

Estas actividades se pueden articular a aspectos tales como:

- Materiales que permiten evidenciar mayor fuerza de atracción.

- Construcción de detectores más sensibles.

- Diferencias entre este fenómeno y aquellos que también se caracterizan por exhibir efectos atractivos. 


\section{Exploración de materiales y fuerzas de atracción}

Haciendo un reconocimiento del trabajo de Gilbert (1600) para diferenciar entre los fenómenos eléctricos y los magnéticos, se encuentra fundamental hacer un trabajo de exploración del comportamiento de distintos materiales respecto a la frotación. Un trabajo de experimentación cualitativa con materiales de uso común como vidrio, pasta, plástico, acetato, metal, o materiales de uso frecuente en laboratorios como ebonita o ámbar y comparar la fuerza de atracción que ellos manifiestan cuando son frotados con otro material (como paño, lino, seda, entre otros) permite hacer una primera organización de los materiales de acuerdo a la forma como evidencian electrificación, por ejemplo al acercarlos a un objeto pequeño y sensible (detector). Se asume como efecto fundamental para evidenciar electrificación, el efecto de atracción. En la presentación usual no se tipifica ningún tipo de efecto, se menciona la atracción y la repulsión para diferenciar tipos de electricidad. A partir de esta experiencia básica es posible avanzar en la organización en torno al comportamiento de los materiales con preguntas relacionadas con la evidencia de electrificación:

¿Se manifiesta la electrificación en todos los materiales, sin importar con cuál se han frotado previamente? ¿̇ solamente experimentan electrificación algunos materiales?

¿Podrían organizarse los materiales de acuerdo con su grado de electrificación? ¿Cuál sería el orden que se pondría y cuáles serían los criterios para hacerlo?

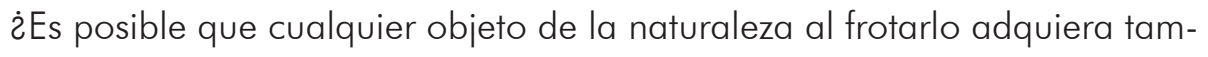
bién la virtud de atraer?

¿Se afecta el grado de atracción si el material con el que se frota el objeto cambia; por ejemplo, vidrio frotado con paño y vidrio frotado con seda?

Estas preguntas orientan el proceso de experimentación y resultan significativas para empezar porque permiten explorar cómo se comportan los materiales al frotarlos. Se hace un reconocimiento del material con que se frota, aspecto que suele ignorarse. Así, a partir de cuestionamientos como estos, es posible construir por ejemplo un cuadro donde se registren los efectos observados al frotar distintos materiales.

Como materiales se puede sugerir vidrio, pasta, ebonita, corcho, acetato, que a su vez se frotan con otros materiales como seda, paño, lino, algodón o papel. Se observa en el detector un comportamiento que corresponde a fuerza de atracción que puede ser excelente, buena, aceptable o mala. Esto da la posibilidad de hacer una primera organización del comportamiento de los materiales en un orden construido que puede ser primero, segundo y tercer orden.

Para avanzar en la organización del fenómeno se consideran relevantes 
¿El grado de electrificación del material depende del frotamiento? Esto es, ¿̇mayor fuerza de frotamiento garantiza mayor electrificación? (Pregunta que a propósito se tuvo en cuenta en la fase de exploración de las explicaciones con los estudiantes)

¿Para observar electrificación siempre es necesario frotar? $\bigcirc$ żes posible observar electrificación solamente por el contacto entre dos materiales?

¿Qué se espera que suceda si se frota un material consigo mismo, por ejemplo, vidrio con vidrio?

¿En qué medida el material con que se frota afecta el registro? Al frotar metal con cualquier otro material, ¿̇qué ocurre con el grado de atracción?

Se espera que con este tipo de experimentación se puedan hacer relevante para los estudiantes aspectos como los siguientes:

1. En principio, todos los materiales de la naturaleza se pueden electrificar. No obstante, unos lo hacen mejor que otros.

2. El mayor o menor grado de atracción depende de los materiales que se frotan entre sí y no de la frotación misma.

3. Los metales requieran de ciertas condiciones (como puede ser estar asilados) para poder registrar efectos observables.

4. Se puede obtener una selección de los mejores materiales, cuyo grado de atracción sea el mayor posible para utilizarlos posteriormente.

Con el trabajo anterior (expresado tal vez mediante un taller) se abre todo un abanico de posibilidades de indagación sobre el comportamiento de los materiales, extraer a partir de la experiencia conclusiones que sean significativas, enriquecer la experiencia sensible del estudiante, empezar a organizar una imagen en torno a lo electrostático, posibilitar explicaciones ligadas a la experiencia misma y contrastar su conocimiento del fenómeno con otras formas de conocimiento tradicionalmente aceptadas.

Es importante mostrar que en la presentación que hacen usualmente los libros analizados no se considera relevante explorar el comportamiento de los materiales. En ellos se asume tácitamente que existen dos tipos de carga eléctrica en la naturaleza; aunque suelen utilizar ebonita y ámbar para establecer esta caracterización, no justifican por qué el ámbar es un buen material, o por qué no utilizan metales para ello. Tampoco suelen mencionar con qué otro material se frotó, ya que para este tipo de presentaciones el material con que se frota no cuenta.

\section{Detectores sensibles}

Usualmente, en los laboratorios se considera como detector el electroscopio. Sin embargo, en la experimentación cualitativa surge como necesidad, para obtener cada vez mejores registros o grados de electrificación, la exploración de materiales que cumplan requisitos de ser livianos y de fácil movilidad de giro. El problema de qué hacer para que el efecto de atracción sea mayor, ya sea para establecer diferencias entre ellos o para registrar efectos más débiles, tiene implicaciones teórico-prácticas. Faraday destaca que en cualquier situación "el cuerpo que atrae es también atraído" (1843), lo cual implica explorar si todos los materiales se dejan atraer y qué materiales pueden ser atraídos con una mayor intensidad. De esta manera se puede avanzar en la comprensión de lo que significa la electrificación de los cuerpos. Implica también explicitar los criterios que han de tenerse en cuenta en 
su disposición para lograr un mayor efecto y que, por ende, deben orientar el diseño del detector.

Un ejemplo sencillo de detector lo planteó Faraday al emplear una fina hebra de hilo metálico suspendida. Luego llegó a utilizar los propios materiales frotados al suspenderlos de un estribo de papel, lo que le da movilidad horizontal. También resalta cómo obtener soportes que permitan una mejor movilidad: el vidrio convexo de un reloj, tiras de papel en forma de aro, entre otros.

Para nuestro caso es importante prestar atención a la construcción de detectores que tengan bastante sensibilidad y permitan obtener grados de atracción excelentes. Un ejemplo puede ser diseñar y construir el Versorium de Gilbert.

Se espera que como consecuencia de esta exploración se llegue de manera natural y organizada a la utilización de electrómetros y electroscopios para detectar y medir electrificación.

A partir de esta exploración se puede concluir que los siguientes aspectos son relevantes:

1. Los metales son los que resultan mejor atraídos (por ejemplo, un fino hilo metálico).

2. Mejorar las condiciones de movilidad lineal y de torsión permite hacer buenos detectores.

3. Se puede avanzar en un proceso de calibración para transformarlos en instrumentos de medida.

Finalmente, y a partir de esta actividad experimental, orientada por preguntas, es posible llenar de sentido los fenómenos asociados a la electrostática y construir explicaciones afines al comportamiento de los materiales, identificando como importante la actividad del medio, propio de la teoría de campos. En este sentido otros núcleos de actividades pueden estar en fenómenos como: la conservación de la electrificación, la comunicación de la electrificación (conductora y aislante), la inducción eléctrica - inducción eléctrica. Sobre la cuantificación de la electrificación (cuantificación de la carga).

\section{Conclusiones}

Las dificultades que evidencian los estudiantes para explicar fenómenos electrostáticos guardan relación directa con la retórica de los textos analizados. Las plantillas que se les aplicaron permiten concluir que tienen en común el modelo de ciencia desde el cual se hacen afirmaciones y se establecen definiciones. Los estudiantes dan explicaciones desde una visión de partículas, según la cual la acción de la carga eléctrica está centrada en los electrones. Sin embargo, este enfoque presenta dificultades. Por una parte, se considera que la carga eléctrica se encuentra concentrada en el propio electrón; cuando este se transfiere de un 
cuerpo a otro, lleva la carga eléctrica consigo, pero como no hay límite en la transferencia de electrones entonces tampoco hay límite en la capacidad de atracción; de ahí que afirmen que "aumentan las cargas negativas, entonces aumenta la fuerza de atracción". Por otra parte, se cree que la carga se debe a la excitación de los electrones, es su movimiento el que determina la carga. No es un problema de transferencia sino de alteración, algo así como un vitalismo que hace que al frotar con más intensidad aumente el movimiento de los electrones y por lo tanto también la fuerza de atracción. Estas respuestas no se corresponden con lo que sucede. Por su parte, los libros de texto analizados basan sus definiciones y explicaciones en el modelo de partículas o en la llamada estructura atómica de la materia. Se considera la transferencia de electrones de un cuerpo a otro como la causa de que un cuerpo cargue eléctricamente, pero no hacen referencia a límites en el proceso de transferencia. Hay información diferente sobre el carácter de la carga eléctrica, lo cual promueve imágenes distorsionadas del fenómeno eléctrico en los estudiantes. Ninguno de los textos analizados, ni los estudiantes, tienen en cuenta el medio (campo) en sus explicaciones.

Un segundo aspecto que se hace relevante tiene que ver con el tipo de experiencias y experimentos que presentan los textos así como con las definiciones y explicaciones que ofrecen, y que se acomodan al argumento teórico que quieren mostrar. Al introducir el concepto de carga eléctrica, por ejemplo, se suele acudir a los efectos de atracción y de repulsión entre cuerpos electrificados por frotación. La experiencia realizada se presenta como si se acomodara directa y perfectamente con el enunciado de que los cuerpos poseen carga eléctrica, la cual es positiva y negativa, y que cargas iguales se repelen y contrarias se atraen. Se omiten los presupuestos que son base de la organización de la experiencia sensible y no se plantean, preguntas como las que se hacen en el cuestionario para los estudientes. En estas formas de presentación de la experiencia no se distingue -como les decía Faraday a sus contemporáneos-entre la hipótesis y el hecho, entre el enunciado teórico y la experiencia sensible, entre la organización y lo organizado, para darle de esta manera un carácter realista a las teorías. Es entendible que ello ocurra, dada la intencionalidad que anima a la mayoría de los textos: presentar los resultados, los puntos de llegada. Una retórica dogmática o magistral. Por su parte, la dificultad que tienen los estudiantes con las preguntas del cuestionario y el buscar explicaciones en la "transferencia de electrones" forma parte de esta manera de pensar la ciencia. Si bien la construcción histórica de la teoría electromagnética se basa en una abundante experiencia, como se comentó anteriormente, el referente de los estudiantes es escaso, tanto que desconocen por completo los experimentos mas significativos sobre cuantificación de la carga. Además, los experimentos que se presentan en el cuestionario son nuevos para ellos, de ahí la confusión en la respuesta. No muestran capacidad de utilizar referentes teóricos coherentes. Su formación en la ciencia es dogmática.

Un tercer aspecto que se hace relevante es la poca importancia que dan los libros analizados a la historia del electromagnetismo. Algunos de ellos lo abordan con un enfoque histórico, así: se presenta una descripción biográfica del personaje cuyo aporte a la electricidad es importante. En este sentido, y por tradición, se describe la vida y obra de científicos como Franklin, Coulomb, Ampere, Faraday y Maxwell, así como sus aportes y no más. No se reconoce la actividad experimental genuina en sus investigaciones, como sugiere Artigas (1989). Es claro que la imagen de 
historia que presentan los textos mencionados corresponde a una descripción de tipo cronológico y secuencial del desarrollo de la ciencia. Si bien esta ubica espacio-temporalmente a la persona, no permite conocer las dificultades y problemas que tuvieron los científicos en la época en que vivieron. Este tipo de historia es criticada por los historiadores modernos, entre ellos Kuhn (1962), para quien los libros de texto tergiversan el sentido de la historia y hacen que se maneje una imagen de ciencia como una constelación de hechos, teorías y métodos y que no se corresponde con las preocupaciones e intereses que han movido el conocimiento científico a lo largo de su desarrollo. La historia del electromagnetismo es diferente a la forma como la pretenden mostrar los textos analizados. Hay dos visiones claramente diferentes, tanto epistemológica como conceptualmente: la visión de partículas y la visión de campos, sin embargo los textos no hacen explícita esta diferencia, lo cual promueve la confusión en el estudiante. Los autores son conscientes de que, al final, lo que importan son los problemas matemáticos.

Por último, es posible pensar los libros de manera diferente, acudiendo a una retórica que promueva una imagen de la ciencia como actividad cultural, en la que los estudiantes y profesores puedan organizar los fenómenos, realizar actividad experimental significativa y construir explicaciones contrastando argumentos.

\section{Referencias}

Alambique (1997). Monográfico: Libros de texto, 11. Barcelona: Editorial Graó.

Artigas, M. (1989). Filosofía de la ciencia experimental; introducción. Ediciones Universidad de Navarra. España.

Calderero, J. F. (2003). Estudio sobre libros de texto de ciencias de la naturaleza mediante análisis cuantitativo basado en la teoría de grafos (tesis de doctorado). Universidad Complutense de Madrid. España.

Del Carmen, L. y Jiménez, M. (1997). Los libros de texto: un recurso flexible. Alambique, $11,7-32$.

Faraday, M. (1843). Sobre la acción inductiva estática [carta enviada a Philips, R.]. Philosophical Magazine, 22.

Furió, C. (1997). Deficiencias epistemológicas en la enseñanza habitual de los conceptos de campo y potencial electrico. Enseñanza de las Ciencias, 15(2), 259-271.

García, E. G. (1999). Construcción del fenómeno eléctrico en la perspectiva de campos. Elementos para una ruta pedagógica (tesis de maestría). Universidad Pedagógica Nacional, Bogotá, Colombia.

García, E. G. (201 1). Las prácticas experimentales en los textos y su influencia en el aprendizaje: aporte histórico y filosófico en la física de campos (tesis doctoral). 
Universidad Autónoma de Barcelona, Barcelona, España.

Gilbert, W. (1600). De Magnete. Limusa.

Guidoni P., Arca, M. y Mazzolli, P. (1990). Enseñar ciencia. Cómo empezar: reflexiones para una educación científica de base. Barcelona: Paidós.

Izquierdo, M. (2005). La función retórica de las narraciones en los libros de texto. Enseñanza de las Ciencias, núm. extra, VII congreso.

Izquierdo, M. (2005a). Estructuras retóricas en los libros de ciencias. Tarbinya. Revista de Investigación e Innovación Educativa, 36, $11-34$.

Izquierdo, M. y Ribera, L. (1997). La estructura y la comprensión de los textos de ciencias. Alambique, 11, 24-33.

Kuhn, T. (1962). La estructura de las revoluciones científicas. Ediciones del Fondo de Cultura Económica, Breviarios.

Maxwell, J. C. (1951). A treatise of electricity and magnetism (vol. 1). Nueva York: Dover.

Moreira, M. A.; Greca, I. M. (1998). Modelos mentales y aprendizaje de Física en electricidad y magnetismo. Enseñanza de las Ciencias, 16, 289-303.
Ogborn, J.; Kress, G. y Martins, I. (1998). Formas de explicar: la enseñanza de las ciencias en secundaria. Madrid: Santillana.

Otero, J. (1990). Variables cognitivas y metacognitivas en la comprensión de los libros de texto. Enseñanza de las Ciencias, 8(1), 17-22.

Perales, F. y Jiménez, J (2002). Las ilustraciones en la enseñanza-aprendizaje de las ciencias. Análisis de libros de texto. Enseñanza de las Ciencias, 20(3), 369-386.

Solbes, J. y Furio, C. (2005). Visiones distorsionadas de la ciencia en los libros de texto. Enseñanza de las Ciencias, núm. extra, VII Congreso.

\section{Para citar este artículo}

García, G. (2017). Imágenes de ciencia en los textos universitarios: aportes para nuevas retóricas desde la actividad experimental en electrostática. Revista de la Facultad de Ciencia y Tecnología-Tecné, Episteme y Didaxis, TED, 41, 149-167. 